\title{
ELASTOPLASTIC STABILITY OF THIN RECTANGULAR PLATES UNDER COMPLEX AND IMPURE LOADING
}

\author{
VU CONG HAM \\ Le Quy don Technical University
}

\begin{abstract}
This paper deals with investigation of the elastoplastic stability of thin rectangular plates. The plate considered herein is subjected to the biaxial compressive forces which are assumed to be linearly distributed along every its edge.

The governing equations of the problem are formulated with applying the elastoplastic process theory whereas Bubnov - Galerkin method is used to calculate the critical forces.

In the paper the author proposes a new method to determine the elements of the matrix concerned with the instability moment of the structure and applies the Gaussian quadric method for integral calculation. Some results of numerical calculations are also presented in the paper.
\end{abstract}

\section{Introduction}

Let's consider a thin rectangular plate which has the biaxial dimensions $a, b$ and the thickness $h$. A coordinate orthogonal system $O x y z$ (or $O x_{1} x_{2} x_{3}$ in tensor notations) is attached to the plate so that the plane $O x y$ coincides with the middle surface and the four edges can be mathematically described as $x=0, y=0, x=a$, $y=b$, respectively.

In $[2,3,4,5]$. the so called pure loaded state is considered. According to this loaded state, the plate is subjected to one or any combination of biaxial compressive forces $p, q$ and shear force $\tau$ (figure 1). These external forces are assumed to act in the middle surface and to be evenly distributed along every edge of the plate. Because of this, the prebuckling stress-strain state is pure at any point in the plate

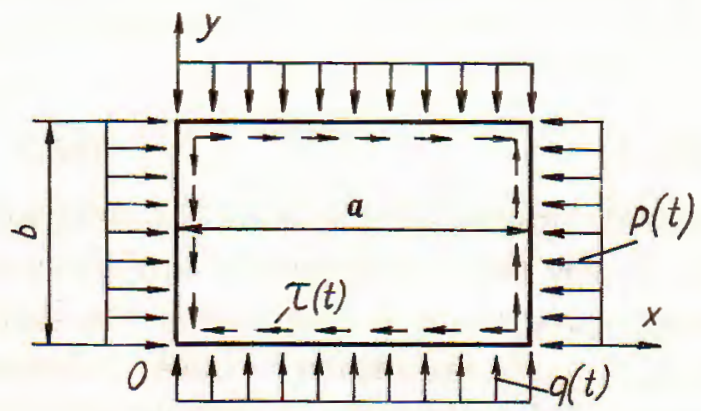

Fig. 1 
This paper is concerned with the impure loading. The plate in the considered case is subjected to biaxial compressive forces $p, q$ which are also assumed to act in the middle surface, but to be unevenly distributed along each edge, respectively. Because of mathematical difficulties, the expressions of $p$ and $q$ are not general, but accepted to be the product of two functions as follows

$$
p=p_{1}(t) \cdot p_{2}(y), \quad q=q_{1}(t) \cdot q_{2}(x)
$$

where $p_{1}(t), q_{1}(t)$ can be called as the process functions which depend only on the process parameter $t$ and increase with respect to the increment of $t ; p_{2}(y), q_{2}(x)$ are called as the distribution functions which characterize the dependence of $p$ and $q$ on the coordinates. According to the form of the distribution functions $p_{2}(y), q_{2}(x)$, the problem can be considered in three cases:

a) $p_{2}(y)=q_{2}(x) \equiv 1$ :

$$
p=p_{1}(t), \quad q=q_{1}(t)
$$

the loading (1.1) returns to the pure loading without shear force $\tau$.

b) $p_{2}(y), q_{2}(x)$ are linear

$$
p=p_{1}(t) \cdot\left(a_{1} y+b_{1}\right), \quad q=q_{1}(t) \cdot\left(a_{2} x+b_{2}\right)
$$

(where $a_{1}, b_{1}, a_{2}, b_{2}$ are constant) the loading can be called as the linear loading (figure 2)

c) $p_{2}(y), q_{2}(x)$ are arbitrarily continuous, the loading can be called as the curve loading (figure 3)

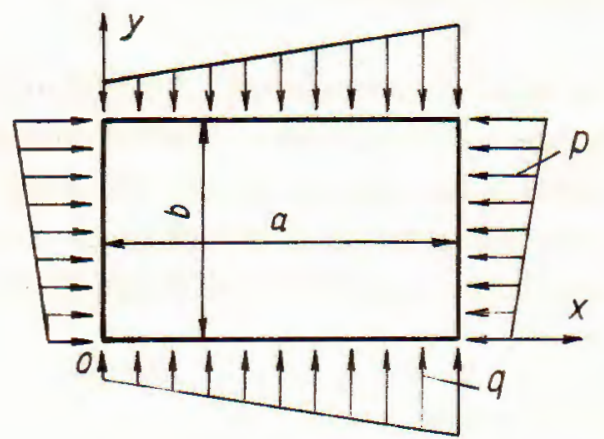

Fig. 2

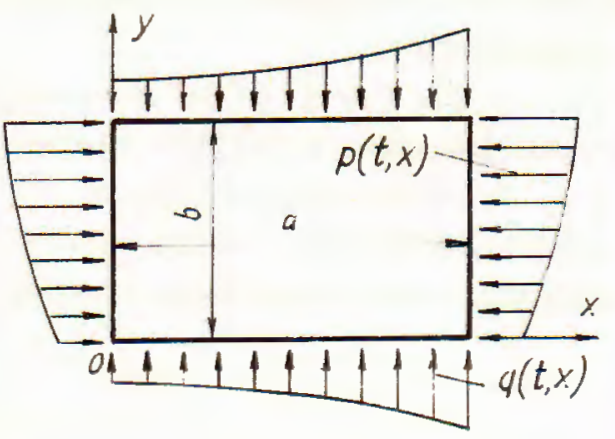

Fig. 3

Because the loading is assumed to be known, the functions $p_{1}(t), q_{1}(t), p_{2}(y)$, $q_{2}(x)$ are known as well. In the fact of solving the problem on PC, these functions are always chosen before the programme is performed. The loading is really complex if the process functions $p_{1}(t), q_{1}(t)$ are chosen in linear independence.

The governing equations of the problem are formulated with applying the theory of the elsatoplastic process [1]. Some results received from solving numerically the problem are also given out in this paper. 


\section{Stability of rectangular plates subjected to linear loading}

\subsection{Governing equations of the problem}

\subsubsection{Prebuckling stage}

Let's consider an arbitrary point $M(x, y, z)(0 \leq x \leq a, 0 \leq y \leq b,-h / 2 \leq z \leq$ $h / 2)$ in the plate. At any moment $t$ in the prebuckling stage, there exists a plane stress state

$$
\begin{aligned}
& \sigma_{11}=-p_{1}(t) \cdot\left(a_{1} y+b_{1}\right) \equiv-p, \\
& \sigma_{22}=-q_{1}(t) \cdot\left(a_{2} x+b_{2}\right) \equiv-q, \\
& \sigma_{12}=\sigma_{23}=\sigma_{13}=\sigma_{33}=0,
\end{aligned}
$$

so that

$$
\sigma=\frac{\sigma_{11}+\sigma_{22}}{3}, \quad \sigma_{u}=\sqrt{\sigma_{11}^{2}-\sigma_{11} \sigma_{22}+\sigma_{22}^{2}} .
$$

The stress components (2.1) satisfy the equilibrium equations, the boundary conditions of the plate and the Beltrami's equation of continuity.

The corresponding components of deformation velocity are determined according to the theory of elastoplastic process [1]

$$
\begin{aligned}
& \dot{\varepsilon}_{11}=\frac{1}{A}\left(\dot{\sigma}_{11}-\frac{1}{2} \dot{\sigma}_{22}\right)+\left(\frac{1}{P}-\frac{1}{A}\right) \frac{\sigma_{11} \dot{\sigma}_{11}+\sigma_{22} \dot{\sigma}_{22}-\frac{1}{2} \sigma_{11} \dot{\sigma}_{22}-\frac{1}{2} \sigma_{22} \dot{\sigma}_{11}}{\sigma_{u}^{2}}\left(\sigma_{11}-\frac{1}{2} \sigma_{22}\right) \\
& \dot{\varepsilon}_{22}=\frac{1}{A}\left(\dot{\sigma}_{22}-\frac{1}{2} \dot{\sigma}_{11}\right)+\left(\frac{1}{P}-\frac{1}{A}\right) \frac{\sigma_{11} \dot{\sigma}_{11}+\sigma_{22} \dot{\sigma}_{22}-\frac{1}{2} \sigma_{11} \dot{\sigma}_{22}-\frac{1}{2} \sigma_{22} \dot{\sigma}_{11}}{\sigma_{u}^{2}}\left(\sigma_{22}-\frac{1}{2} \sigma_{11}\right) \\
& \varepsilon_{12}=\varepsilon_{23}=\varepsilon_{31}=0, \quad \varepsilon_{33}=-\left(\varepsilon_{11}+\varepsilon_{22}\right)
\end{aligned}
$$

where, for the processes of average curvature

$$
A=\frac{\sigma_{u}}{s}, \quad P=\Phi^{\prime}(s), \quad \frac{d s}{d t}=\frac{2}{\sqrt{3}}\left(\dot{\varepsilon}_{11}^{2}+\dot{\varepsilon}_{11} \dot{\varepsilon}_{22}+\dot{\varepsilon}_{12}^{2}\right)^{1 / 2}
$$

$\Phi^{\prime}(s)$ - a known function concerned with the material used, $s$ - the arc-length of the strain trajectory. The mark (.) is derivative of the corresponding quantity with respect to the process parameter $t$.

Solving the system of differential equation $(2.2),(2.3)$ in the combination with the initial conditions of the problem one can determine the stress-strain state at any point $M$ in the plate at any moment of the prebuckling stage.

2.1.2. Postbuckling stage

Let $t$ increase until it reaches the value $t=t^{*}$ at which a bifurcation of equilibrium states appears. It means: with an infinitesimal small increment of the external forces 
there are possible increments of deformation (including the bending deformation) in the plate.

Suppose deformation increments of the middle surface are equal to zero at the moment the instability appears. According to the assumption of straight normal, we can get the increments of deformation at the considered point $M$

$$
\delta \varepsilon_{i j}=-z \delta \chi_{i j}=-z \frac{\partial^{2} \delta w}{\partial x_{i} \partial x_{j}}
$$

where

$\delta \chi_{i j}=\frac{\partial^{2} \delta w}{\partial x_{i} \partial x_{j}}$ - increments of curvature and torsion associated with instability,

$\delta w$ - deflection increment of the middle surface.

The corresponding stress increments can be determined according to the theory of the elastoplastic process

$$
\begin{gathered}
\delta \sigma_{i j}=\frac{2}{3} A\left(\delta \varepsilon_{i j}+\delta_{i j} \delta \varepsilon_{k k}\right)+(P-A) \frac{\sigma_{k \ell} \delta \varepsilon_{k \ell}}{\sigma_{u}^{2}} \sigma_{i j}, \\
\delta s=\frac{2}{\sqrt{3}}\left(\delta \varepsilon_{11}^{2}+\delta \varepsilon_{11} \delta \varepsilon_{22}+\delta \varepsilon_{22}^{2}\right)^{1 / 2} \\
\quad(i, j, k, \ell=1,2)
\end{gathered}
$$

The increments of membrane forces and bending moments are determined as

$$
\delta N_{i j}=\int_{-h / 2}^{h / 2} \sigma_{i j} d z, \quad \delta M_{i j}=\int_{-h / 2}^{h / 2} \sigma_{i j} z d z .
$$

Using (2.4), (2.5) and note that $A$ and $P$ do not depend on $z$ in the case that the increments of the middle surface are absent [2], we reach

$$
\begin{aligned}
\delta N_{i j} & =-\left[\frac{2}{3} A\left(\frac{\partial^{2} \delta w}{\partial x_{i} \partial x_{j}}+\delta_{i j} \frac{\partial^{2} \delta w}{\partial x_{k} \partial x_{k}}\right)+(P-A) \frac{\sigma_{i j} \sigma_{k l}}{\sigma_{u}^{2}} \cdot \frac{\partial^{2} \delta w}{\partial x_{k} \partial x_{\ell}}\right] \int_{-h / 2}^{h / 2}=0 \\
\delta M_{i j} & =-\left[\frac{2}{3} A\left(\frac{\partial^{2} \delta w}{\partial x_{i} \partial x_{j}}+\delta_{i j} \frac{\partial^{2} \delta w}{\partial x_{k} \partial x_{k}}\right)+(P-A) \frac{\sigma_{i j} \sigma_{k \ell}}{\sigma_{u}^{2}} \cdot \frac{\partial^{2} \delta w}{\partial x_{k} \partial x_{\ell}}\right] \int_{-h / 2}^{h / 2} z^{2} d x \\
& =-\left[\frac{2}{3} A\left(\frac{\partial^{2} \delta w}{\partial x_{i} \partial x_{j}}+\delta_{i j} \frac{\partial^{2} \delta w}{\partial x_{k} \partial x_{k}}\right)+(P-A) \frac{\sigma_{i j} \sigma_{k \ell}}{\sigma_{u}^{2}} \cdot \frac{\partial^{2} \delta w}{\partial x_{k} \partial x_{\ell}}\right] \frac{h^{3}}{12}
\end{aligned}
$$


or in the development form

$$
\begin{aligned}
& \delta M_{11}=-\frac{G h^{3}}{4}\left(R_{1} \frac{\partial^{2} \delta w}{\partial y^{2}}+R_{2} \frac{\partial^{2} \delta w}{\partial y^{2}}\right) \\
& \delta M_{12}=\delta M_{21}=-\frac{G h^{3}}{4} \cdot R_{3} \frac{\partial^{2} \delta w}{\partial x \partial y} \\
& \delta M_{22}=-\frac{G h^{3}}{4}\left(R_{4} \frac{\partial^{2} \delta w}{\partial x^{2}}+R_{5} \frac{\partial^{2} \delta w}{\partial y^{2}}\right)
\end{aligned}
$$

where

$$
\begin{aligned}
R_{1} & =\frac{4}{3} \varphi_{A}+\left(\varphi_{P}-\varphi_{A}\right) \hbar, \\
R_{2} & =\frac{2}{3} \varphi_{A}+\left(\varphi_{P}-\varphi_{A}\right) \rho, \\
R_{3} & =\frac{2}{3} \varphi_{A}, \\
R_{4} & =\frac{2}{3} \varphi_{A}+\left(\varphi_{P}-\varphi_{A}\right) \rho, \\
R_{5} & =\frac{4}{3} \varphi_{A}+\left(\varphi_{P}-\varphi_{A}\right) \lambda, \\
\hbar & =\frac{\sigma_{11}^{2}}{\sigma_{u}^{2}}, \quad \rho=\frac{\sigma_{11} \sigma_{22}}{\sigma_{u}^{2}}, \quad \lambda=\frac{\sigma_{22}^{2}}{\sigma_{u}^{2}}, \quad \varphi_{A}=\frac{A}{3 G}, \quad \varphi_{P}=\frac{P}{3 G}
\end{aligned}
$$

Now the stability equation of the plate

$$
\frac{\partial^{2} \delta M_{i j}}{\partial x_{i} \partial x_{j}}+N_{i j} \delta \chi_{i j}=0
$$

becomes

$$
\begin{gathered}
R_{1} \frac{\partial^{4} \delta w}{\partial x^{4}}+\left(R_{2}+2 R_{3}+R_{4}\right) \frac{\partial^{4} \delta w}{\partial x^{2} \partial y^{2}}+R_{5} \frac{\partial^{4} \delta w}{\partial y^{4}}+2 \frac{\partial R_{1}}{\partial x} \cdot \frac{\partial^{3} \delta w}{\partial x^{3}}+ \\
+2 \frac{\partial\left(R_{3}+R_{4}\right)}{\partial y} \cdot \frac{\partial^{3} \delta w}{\partial x^{2} \partial y}+2 \frac{\partial\left(R_{2}+R_{3}\right)}{\partial x} \cdot \frac{\partial^{3} \delta w}{\partial x \partial y^{2}}+2 \frac{\partial R_{5}}{\partial y} \frac{\partial^{3} \delta w}{\partial y^{3}}+ \\
\left(\frac{\partial^{2} R_{1}}{\partial x^{2}}+\frac{\partial^{2} R_{4}}{\partial y^{2}}-\frac{4 \sigma_{11}}{G h^{2}}\right) \frac{\partial^{2} \delta w}{\partial x^{2}}+2 \frac{\partial^{2} R_{3}}{\partial x \partial y} \cdot \frac{\partial^{2} \delta w}{\partial x \partial y}+\left(\frac{\partial^{2} R_{2}}{\partial x^{2}}+\frac{\partial^{2} R_{5}}{\partial y^{2}}-\frac{4 \sigma_{22}}{G h^{2}}\right) \frac{\partial^{2} \delta w}{\partial y^{2}}=0
\end{gathered}
$$

or

$$
\begin{aligned}
\alpha_{1} \frac{\partial^{4} \delta w}{\partial x^{4}} & +\alpha_{2} \frac{\partial^{4} \delta w}{\partial x^{2} \partial y^{2}}+\alpha_{3} \frac{\partial^{4} \delta w}{\partial y^{4}}+\alpha_{4} \frac{\partial^{3} \delta w}{\partial x^{3}}+\alpha_{5} \frac{\partial^{3} \delta w}{\partial x^{2} \partial y}+\alpha_{6} \frac{\partial^{3} \delta w}{\partial x \partial y^{2}}+ \\
& +\alpha_{7} \frac{\partial^{3} \delta w}{\partial y^{3}}+\alpha_{8} \frac{\partial^{2} \delta w}{\partial x^{2}}+\alpha_{9} \frac{\partial^{2} \delta w}{\partial x \partial y}+\alpha_{10} \frac{\partial^{2} \delta w}{\partial y^{2}}=0
\end{aligned}
$$


where

$$
\begin{gathered}
\alpha_{1}=R_{1}=\frac{4}{3} \varphi_{A}+\left(\varphi_{P}-\varphi_{A}\right) \hbar \\
\alpha_{2}=\left(R_{2}+2 R_{3}+R_{4}\right)=\frac{8}{3} \varphi_{A}+2\left(\varphi_{P}-\varphi_{A}\right) \rho \\
\alpha_{3}=R_{5}=\frac{4}{3} \varphi_{A}+\left(\varphi_{P}-\varphi_{A}\right) \lambda \\
\alpha_{4}=2 \frac{\partial R_{1}}{\partial x}=\frac{8}{3} \frac{\partial \varphi_{A}}{\partial x}+2 \frac{\partial\left(\varphi_{P}-\varphi_{A}\right)}{\partial x} \hbar+2\left(\varphi_{P}-\varphi_{A}\right) \frac{\partial \hbar}{\partial x}, \\
\alpha_{5}=2 \frac{\partial\left(R_{3}+R_{4}\right)}{\partial y}=\frac{8}{3} \frac{\partial \varphi_{A}}{\partial y}+2 \frac{\partial\left(\varphi_{P}-\varphi_{A}\right)}{\partial y} \rho+2\left(\varphi_{p}-\varphi_{A}\right) \frac{\partial \rho}{\partial y} \\
\alpha_{6}=2 \frac{\partial\left(R_{3}+R_{2}\right)}{\partial x}=\frac{8}{3} \frac{\partial \varphi_{A}}{\partial x}+2 \frac{\partial\left(\varphi_{P}-\varphi_{A}\right)}{\partial x} \rho+2\left(\varphi_{P}-\varphi_{A}\right) \frac{\partial \rho}{\partial x} \\
\alpha_{7}=2 \frac{\partial R_{5}}{\partial y}=\frac{8}{3} \frac{\partial \varphi_{A}}{\partial y}+2 \frac{\partial\left(\varphi_{P}-\varphi_{A}\right)}{\partial y} \lambda+2\left(\varphi_{P}-\varphi_{A}\right) \frac{\partial \lambda}{\partial y} \\
\alpha_{8}=\frac{\partial^{2} R_{1}}{\partial x^{2}}+\frac{\partial^{2} R_{4}}{\partial y^{2}}-\frac{4 \sigma_{11}}{G h^{2}} \\
=\frac{\partial^{2}}{\partial x^{2}}\left[\frac{4}{3} \varphi_{A}+\left(\varphi_{P}-\varphi_{A}\right) \hbar+\frac{\partial^{2}}{\partial y^{2}}\left[\frac{2}{3} \varphi_{A}+\left(\varphi_{P}-\varphi_{A}\right) \rho\right]-\frac{4 \sigma_{11}}{G h^{2}}\right. \\
\alpha_{9}=2 \frac{\partial^{2} R_{3}}{\partial x \partial y}=\frac{4}{3} \frac{\partial^{2} \varphi_{A}}{\partial x \partial y}, \\
\alpha_{10}=\frac{\partial^{2} R_{2}}{\partial x^{2}}+\frac{\partial^{2} R_{5}}{\partial y^{2}}-\frac{4 \sigma_{22}}{G h^{2}}\left[\frac{2}{3} \varphi_{A}+\left(\varphi_{P}-\varphi_{A}\right) \rho\right]+\frac{\partial^{2}}{\partial y^{2}}\left[\frac{4}{3} \varphi_{A}+\left(\varphi_{P}-\varphi_{A}\right) \lambda\right]-\frac{4 \sigma_{22}}{G h^{2}}
\end{gathered}
$$

\subsection{Determining the critical forces by using Bubnov-Galerkin method}

\subsubsection{The Bubnov-Galerkin method}

The values of external forces at the moment $t=t^{*}$ when the instability occurs are called the critical forces. To determine the critical forces, we have to determine the stress-strain state of any point in the plate at any moment in the prebuckling stage and solve the stability equation (2.10).

The stability equation (2.10) is a partial differential equation of the fourth order with the coefficients $\alpha_{k}(k=1 \div 10)$ depending on $R_{j}(j=1 \div 5)$ and their upto-the-second-order derivatives. According to (2.9), $R_{j}$ are depend on $\varphi_{A}, \varphi_{P}$, $\hbar$, $\rho, \lambda$, i.e. $R_{j}$ depend on $A, P, \sigma_{11}, \sigma_{22}$ and $\sigma_{u}$. Because $A=\sigma_{u} / s, P=\Phi^{\prime}(s)$, $\sigma_{11}=\sigma_{11}(y), \sigma_{22}=\sigma_{22}(x), \sigma_{u}=\sigma_{u}(x, y)$, where $s$ implicitly depends on $x, y$, so $R_{j}$ also implicitly depend on $x, y$. Because of this, a direct solution to the above stability equation is complicated. 
To reach the critical forces according to Bubnov-Galerkin method, we need to fulfill the following steps.

a) Approximating the expression of $\delta w$ in series

$$
\delta w=\sum_{k=1}^{N} B_{k} \delta w_{k}
$$

where $N$ is the number of terms of the series, $B_{k}$ are coefficients different from $0, \delta w_{k}$ are functions being linearly independent and satisfying the boundary conditions.

In fact, $\delta w_{k}$ are chosen as the product of two functions

$$
\delta w_{k}=X_{k} \cdot Y_{k}=X_{k}(x) \cdot Y_{k}(y)
$$

where $X_{k}=X_{k}(x)$ - a function of $x$ only, $Y_{k}=Y_{k}(y)$ - a function of $y$ only.

b) Putting the expression (2.12) of $\delta w$ into the stability equation (2.10) and using notation $\Omega(\delta w)$ for the left side of the received equation. The result is

$$
\Omega(\delta w)=0
$$

Because of (2.12) we can write

$$
\Omega(\delta w)=\sum_{k=1}^{N} B_{k} \Omega\left(\delta w_{k}\right)
$$

where $\Omega\left(\delta w_{k}\right)$ is the expression one can get by putting $\delta w_{k}$ into left side of the stability equation $(2.10)$, instead of putting $\delta w$. It is clear that both $\Omega\left(\delta w_{k}\right)$ and $\Omega(\delta w)$ depend on $x, y$,

c) Multiplying both side of (2.13) by $\delta w_{i}(i=1,2, \ldots, N)$ and integrating both sides of the received equation all over the volume of the plate. The result is

$$
\int_{0}^{a} \int_{0}^{b} \delta w_{i} \Omega(\delta w) d x d y=0 \Leftrightarrow \sum_{k=1}^{N} B_{k} \int_{0}^{a} \int_{0}^{b} \delta w_{i} \Omega\left(\delta w_{k}\right) d x d y=0 .
$$

d) Letting $i$ be equal to $1,2, \ldots, N$ in turn, we get $N$ equations of the form (2.15), i.e, a system of $N$ linear algebraic equations with the unknowns $B_{1}, B_{2}, \ldots, B_{N}$. This system has the form

$$
\left(C_{i k}\right)\left\{B_{k}\right\}=0
$$

where

$$
\left\{B_{k}\right\}^{T}=\left(B_{1}, B_{2}, \ldots, B_{N}\right)
$$


$\left(C_{i k}\right)$ - an $N$-order square matrix,

$$
C_{i k}=\int_{0}^{a} \int_{0}^{b} \delta w_{i} \Omega\left(\delta w_{k}\right) d x d y .
$$

Because $B_{1}, B_{2}, \ldots, B_{N}$ must be non-trivial solutions of the system (2.16), the condition for this is

$$
\operatorname{det}\left(C_{i k}\right)=0 .
$$

In the process of solving the problem to determine strain - stress state of the plate, the process parameter $t$ is monotonely increased, the condition (2.18) allows to find out the moment at which the instability starts to appear. The values of the external forces at this moment are critical forces.

\subsubsection{Determining $C_{i k}$}

The main difficulty in the approach to the cristal forces belongs to how to calculate the elements of matrix $\left(C_{i k}\right)$ according to (2.17). Because the coefficients $R_{j}$ in (2.11) depend implicity on $x$ and $y$, so $\Omega\left(\delta w_{k}\right)$ and $\Omega(\delta w)$ depend implicitly on $x$ and $y$ as well.

To overcome this difficulty, the author would propose an approximate method with the following operations:

a) Dividing the plane of the plate into $M$ rectangular pieces by nodal lines parallel with the edges, respectively (figure 4).

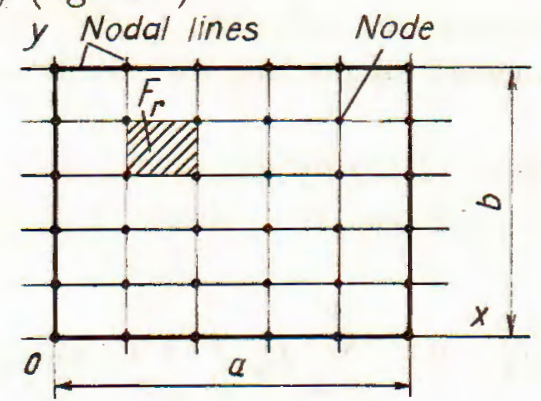

Fig. 4

b) At every vertex (Node) of piece $F_{r}(r=1,2, \ldots, M)$ whose coordinates are known, it is possible to calculate the values of quantities $\varphi_{A}, \varphi_{P}, \hbar, \rho, \lambda$ at any moment $t$.

c) On each piece $F_{r}$ we suppose that $\varphi_{A}, \varphi_{P}, \hbar, \rho, \lambda$ depend linearly on $x, y$

$$
\begin{aligned}
& \varphi_{A}=r_{A 1} x+r_{A 2} y+r_{A 3}, \quad \varphi_{p}=r_{P 1} x+r_{P 2} y+r_{P 3} \\
& \hbar=r_{H 1} x+r_{H 2} y+r_{H 3}, \quad \rho=r_{R 1} x+r_{R 2} y+r_{R 3}, \quad \lambda=r_{L 1} x+r_{L 2} y+r_{L 3}
\end{aligned}
$$

where the coefficients $r_{A 1}, r_{A 2}, r_{A 3}, r_{P 1}, r_{P 2}, r_{P 3}, r_{H 1}, r_{H 2}, r_{H_{3}}, r_{L 1}, r_{L 2}, r_{L 3}$ are determined in accordance with the mifimum square method using the known values 
of the quantities $\varphi_{A}, \varphi_{P}, \hbar, \rho, \lambda$ and the coordinates $x, y$ at the four vertexes of the corresponding piece $F_{r}$.

The assumption (2.19) can be acceptable because of the continuity of the material used and the continuous distribution of the external forces with respect to the coordinates.

d) Putting the determined axpressions of $\varphi_{A}, \varphi_{P}, \hbar, \rho, \lambda$ according to (2.19) into (2.9) to determine $R_{j}$. Afterwards, using (2.11) to determine the coefficients $\alpha_{k}$ $(k=1 \div 10)$ of stability equation $(2.10)$.

e) In equation (2.17) to calculate $C_{i k}$, because $\delta w_{i}, \delta w_{k}$ depend on $x$ and $y$, so it is possible to denote $\delta w_{i} \Omega\left(\delta w_{k}\right)=\Theta_{i k}(x, y)$. Combining with dividing the plane of the plate into $M$ rectangular pieces, we can write

$$
C_{i k}=\sum_{j=1}^{M} \int_{F_{j}} \Theta_{i k}(x, y) d x d y .
$$

f) To implement the integrals in (2.20), we apply the Gaussian quadric method presented in [6]. This method supplies high accuracy results whereas reduces the time expense on PC.

\section{Some results of numerical calculation}

Now we consider a plate made of the stell $30 \mathrm{XГCA}$ which has the shear modulus $G=0.8667 \cdot 10^{6} \mathrm{kG} / \mathrm{cm}^{2}$ and the corresponding material function $\Phi=\Phi(s)$ presented in [1]. Let's accept the geometrical relations as $a / h=55, b / h=45$, and the compressive forces $p, q$ of the form (1.3), where: $p_{1}(t)=2000 t, q_{1}(t)=1500 t^{2}$.

Suppose the plate are simply supported along the four edges, the expression of $\delta w$ which satisfies the boundary conditions is chosen as

$$
\delta w=\sum_{k=1}^{4} \dot{B}_{k} \sin k \pi x a \sin k \pi y b .
$$

Some concrete results are given out in following:

a) If $a_{1}=a_{2}=0, b_{1}=b_{2}=1$ then

$$
p \equiv p_{1}(t)=2000 t, \quad q \equiv q_{1}(t)=1500 t^{2}
$$

i.e. the plate is in the pure loading. In this case, we can determine the critical forces in two ways: one belongs to that of pure loading, the other belongs to the method of the impure loading which is presented in this paper and concerned with dividing the plane of the plate into pieces. Theoretically, the two ways should give the same results.

The performances of the two calculation programmes give us the same results

$$
p^{*}=2452 k G / \mathrm{cm}^{2}, \quad q^{*}=2255 \mathrm{kG} / \mathrm{cm}^{2} .
$$


This proves the soundness of the method proposed in this paper for calculating the elements of matrix $\left(C_{i k}\right)$.

b) If assigning $a_{1}=0, b_{1}=1, a_{2}=1 / a, b_{2}=1$ then we have

$$
p=p_{1}(t)=2000 t, \quad q=1500 t^{2}\left(\frac{x}{a}+1\right) .
$$

The results in this case are $p_{1}^{*}=2104 \mathrm{kG} / \mathrm{cm}^{2}, q_{1}^{*}=1660 \mathrm{kG} / \mathrm{cm}^{2}$.

c) If accepting $a_{1}=0, b_{1}=1, a_{2}=1 / a, b_{2}=0$ then we get

$$
p=p_{1}(t)=2000 t, \quad q=1500 t^{2}\left(\frac{x}{a}\right) .
$$

The performance of the programme gives $p_{1}^{*}=3104 \mathrm{kG} / \mathrm{cm}^{2}, q_{1}^{*}=3613 \mathrm{~kg} / \mathrm{cm}^{2}$.

d) If $a_{1}=1 / b, b_{1}=1, a_{2}=1 / a, b_{2}=0$ then

$$
p=2000 t\left(\frac{y}{b}+1\right), \quad q=1500 t^{2}\left(\frac{x}{a}\right)
$$

We receive $p_{1}^{*}=2380 \mathrm{kG} / \mathrm{cm}^{2}, q_{1}^{*}=2124 \mathrm{kG} / \mathrm{cm}^{2}$.

\section{Conclusion}

In the paper, the author applies the elastoplastic process theory to formulate the governing equation of the stability problem of thin rectangular plates under complex and impure loadings, and gives out some concrete result concerned with calculating the values of critical forces.

When applying the Bubnov-Galerkin method to determining the values of critical forces, the author proposes a possible method to calculate the elements of matrix $\left(C_{i k}\right)$, where the minimum square method and the Gaussian quadric are used.

The author would like to thank Prof. Dao Huy Bich, Hanoi National University and A. Prof. Hoang Xuan Luong, Le Quy Don Technical University for helping the author to complete this paper.

This paper is completed with finalcial support from the National Basic Research Program in National Sciences.

\section{REFERENCES}

1. Dao Huy Bich. Theory of the elastoplastic process. Publishing House of Hanoi National University, 1999.

2. Dao Huy Bich. The loading parameter method in solving elastoplastic stability of thin-walled structures under complex loading. International Conference on Analysis and Mechanics of Continuous Media. Ho Chi Minh City, December $27-29,1995$. 
3. Vu Cong Ham. Elastoplastic stability of thin rectangular plates under complex loadings. Master thesis, Le Quy Don Technical University, 1996 (in Vietnamese).

4. Vu Cong Ham. Solution to the problem of the elastoplastic stability of thin rectangular plates in two cases of boundary condition. Vietnam Journal of Mechanics, NCST of Vietnam, T.XX, 1998, No 4, pages 30-40.

5. Vu Cong Ham. Elastoplastic stability of thin rectangular plates in some different boundary conditions under complex loadings. Proceedings of the Sixth National Conference on Solid Mechanics. Hanoi, 1999, pages 185-193 (in Vietnamese).

6. Barker A. A. The boundary Element Method in Engineering. A complete course. McGraw-Hill Book Company. Printed in Singapore, 1992.

Received August 15, 2001

\section{ỔN ĐỊNH ĐÀN DẺO CƯA TẤM CHŨ̃ NHÂ̂T CHỊU TẢI TRỌNG PHÚC TẠP, KHÔNG THUẦn NHẤT}

Bài báo liên quan đến ổn định đàn dẻo của tấm mỏng chữ nhật chịu tải phức tạp, không thuần nhất. Tải trọng tác dụng lên tấm trong trường hợp đang khảo sát bao gồm các lụ̣c nén theo hai phương phân bố theo quy luật bậc nhất dọc theo các canh của tấm.

Các phương trình giải của bài toán được thiết lập trên cơ sở áp dụng lý thuyết quá trình đàn dẻo. Giá trị tới hạn của các lực ngoài được xác định bằng phương pháp Bubnov - Galerkin.

Để tính các phần tử của ma trận có liên quan đến điều kiện mất ổn định của tấm, tác giả đề xuất phương pháp chia nhỏ mặt phẳng của tấm bằng các đường nút và khào sát trạng thái ứng suất - biến dạng tại các điểm nút tại những thời điểm khác nhau của quá trình. Việc tính các tích phân xuất hiện trong quá trình giải toán được áp dụng phương pháp cầu phương Gauss. Các kết quả tính toán cụ thể bằng số cũng được đưa ra ở trong bài báo. 